Research

\title{
Predictors of long-term function in older community-dwelling people who have presented to an emergency department after a fall: A cohort study
}

Melissa Russell

Department of Epidemiology and Biostatistics, School of Population and Global Health, The University of Melbourne; and National Ageing Research Institute, Parkville, Victoria, Australia

\section{Keith Hill}

School of Physiotherapy and Exercise Science, Curtin University, Perth, Western Australia; and National Ageing Research Institute, Parkville, Victoria, Australia

\section{Lesley Day}

Monash Injury Research Institute, Monash University, Clayton, Victoria, Australia

\section{Teddy Oosterhuis}

Department of Physiotherapy, Utrecht University, Utrecht; and Department of Health Sciences, VU University, Amsterdam, The Netherlands

\section{Irene Blackberry}

National Ageing Research Institute, Parkville; and Department of General Practice, Melbourne Medical School, The University of Melbourne, Parkville, Victoria, Australia

\section{Shyamali C. Dharmage}

Department of Epidemiology and Biostatistics, School of Population and Global Health, The University of Melbourne, Parkville, Victoria, Australia

Aim: To identify factors predictive of function 12 months after a fall and emergency department (ED) presentation. Methods: This was a prospective cohort study with 608 older fallers. After presentation and discharge from the ED, a baseline assessment was initially undertaken and then repeated after 12 months. The Human Activity Profile Adjusted Activity Score (HAP-AAS) at the 12-month follow-up assessment was the functional outcome measure. Results: Over the follow-up period, $37.3 \%(95 \%$ CI 33.4, 41.2) of participants declined in their HAP-AAS score. Increased age, pre-index fall functional impairment, poorer mobility/balance, and sustaining falls and severe injuries over the 12-month follow-up period were some of the factors predictive of a lower HAP-AAS score.

Conclusion: This study highlights the importance of preventing falls in the 12 months after discharge from an $E D$. Some of the factors identified as being predictive of lower function are the same as those previously found to be predictive of falls.

Correspondence to: Dr Melissa Russell, University of Melbourne. Email:melissar@unimelb.edu.au
Key words: accidental falls, activities of daily living, aged, emergency medical services.

\section{Introduction}

Loss of function is a common issue for older people. A recent study found that $34.6 \%$ of people aged 65 and above are dependent in at least one activity of daily living (ADL) [1]. The odds of being dependent in at least one ADL increase with age, with United Kingdom data indicating the prevalence of requiring assistance or having difficulties with ADLs was three and a half times higher in those aged 85 and above compared to those aged 65 to 69 years [2]. The consequences of loss of function are severe and include increased risk of depression [3], residential care admission [4,5] and mortality [6].

Falls, and subsequent injuries, are commonly identified as one of the causes of loss of function [7,8]. Older people who have fallen often presented to emergency departments (EDs) after a fall, with recent studies estimating that $17 \%$ of presentations to EDs by those aged 70 and above are due to falls and that $57 \%$ of these people are discharged directly home [9]. We have previously reported that $35 \%$ of older people who have fallen who were presented to the $\mathrm{ED}$ and were discharged home experienced a decline in function from pre-fall levels at 1 month after their fall [10], while another study reported that $22.7 \%$ still experienced a decline in function three months after their fall [11]. No research has yet been undertaken to investigate the effect of a fall and injury on function one year after a fall in this group of older people who present to an ED.

A post-fall ED presentation has been identified as an opportune time to investigate problems and intervene to prevent further falls and ongoing health-related problems [12-14]. The aim of this study was to identify the factors predictive of function one year after a fall and ED presentation. The clinical implication of this research is that these factors may be used by ED clinicians to identify those older people who will be at risk of lower levels of function one year after the fall and therefore be considered for preventative and rehabilitative services post-ED discharge.

\section{Methods}

Study design and sample

The current study was a cohort study, nested within a randomised controlled trial (RCT) assessing the effectiveness of a falls prevention intervention [15]. The intervention 
group in the RCT received a targeted multifactorial fall prevention program consisting of referrals to existing community services and the control group received the standard care from the ED. No effect from the intervention on falls or injuries was found, therefore for the purpose of this study, participants in the intervention and control group were combined. Written informed consent was obtained from all participants and the Ethics Committees of the participating hospitals approved the RCT.

Participants were recruited from seven acute hospitals in Melbourne, Australia. The ED staff identified potential participants within the ED and through follow-up telephone calls [15]. Inclusion criteria were living in the community, being aged 60 years or older, presenting to an ED after a fall (the index fall), and being discharged home from the ED (i.e. not admitted to the wards). Exclusion criteria were the inability to comply with simple instructions and the inability to walk independently indoors.

\section{Data collection}

Data collection was performed between January 2003 and December 2006.

\section{Baseline assessment data}

Following recruitment, a baseline assessment was conducted in the participant's own home, at a median of 20 days (interquartile range (IQR) 12-33) following the ED presentation. A suite of assessments were undertaken at this time, and at a subsequent home visit assessment 12 months later. Items previously found to be associated with functional decline are reported in this study:

- demographics (age, gender);

- self-report of medical conditions; osteoarthritis, history of stroke, respiratory disease, diabetes mellitus, and Parkinson's disease, recorded with the Falls Risk for Older People in the Community (FROP-Com) assessment tool [16];

- polypharmacy, indicated by taking three or more prescribed medications, recorded with the FROP-Com [16];

- pre-index fall functional impairment, defined as requiring assistance or supervision in personal or domestic ADLs [16];

- fall history (categorised as sustaining only the fall resulting in the presentation to the ED versus that fall plus additional falls in the previous year) [16];

- vision impairment (defined as trouble seeing objects clearly, trouble judging distance, or a history of visual impairment but no vision testing in 2 years, recorded with the FROP-Com;

- body mass index;

- cognitive impairment, indicated by a score of $<7$ on the Abbreviated Mental Test [17];

- function at baseline, measured with the Human Activity Profile (HAP) [18] (see the Outcomes section below for the description of the HAP);
- post-index fall mobility/balance, measured with the Timed Up and Go test (TUG) [19];

- post-index fall depressive symptoms, indicated by a score of $\geq 6$ on the Geriatric Depression Scale-15 [20]; and

- falls efficacy, measured with the Modified Falls Efficacy Scale (MFES) [21]. The MFES was coded into a dichotomous variable around the median, with 0 to 8.5 representing high fear of falling and 8.6 to 10 representing low fear of falling [22].

\section{Index fall injury data}

The index fall was defined as the fall resulting in the initial pre-study recruitment presentation to the ED. Index fall injury data were collected in a review of hospital medical records, with the exception that the records were not available for $9.7 \%$ of participants. For these participants selfreport data, collected in the baseline assessment, was the data source. The index fall injury related factor examined in this study was injury type. Each type of injury was treated as a dichotomous variable so participants could have multiple types of injuries in the index fall (Table 1).

\section{Twelve-month follow-up period data}

Participants were monitored for 12 months after the baseline assessment using a falls diary, regular telephone calls and medical record review. The full description of falls diary data collection methods can be found elsewhere [15]. For the purposes of this study, the falls and injury data were coded into four categories: no falls, one fall without serious injury (Abbreviated Injury Score $\geq 2$ [23]), two or more falls without serious injury, one or more falls with serious injury [24].

\section{Final 12-month assessment data}

At the completion of the 12-month follow-up period, participants were visited at home and the baseline assessments repeated. The outcome measure, function, was measured with the HAP-Adjusted Activity Score (AAS). The HAPAAS assesses the number of activities from a 94-item hierarchically ordered list (in terms of energy expenditure) that an individual can perform, no longer perform or has never performed $[18,25]$. The HAP-AAS can range from 0 to 94 and is the score assigned to the highest level activity an individual can perform minus one point for each lower numbered activity the individual can no longer perform. The 12-month follow-up assessment was performed at a median of 370 days (IQR 365, 378) after the baseline assessment.

\section{Statistical analysis}

To examine the association between the exposures measured in the baseline assessment, index fall data, the 12-month follow-up period and the outcome variable of HAP-AAS score, univariate and multiple linear regression were used. When non-linear associations were found and the assumptions of the linear regression model were not met, the exposure variables were transformed by taking the $\log$ of the 
Table 1: Characteristics of participants and association between potential predictors of long-term function, measured by the HAP-AAS, at the 12-month follow-up assessment (all data $n(\%)$ unless otherwise stated)

\begin{tabular}{|c|c|c|}
\hline Characteristic & $\begin{array}{l}\text { All participants } \\
n=608(\%)\end{array}$ & $\begin{array}{c}\text { Change ( } \beta \text { value) in HAP-AAS at } \\
12-\text {-month follow-up }(95 \% \mathrm{CI})\end{array}$ \\
\hline \multicolumn{3}{|l|}{ Baseline assessment data } \\
\hline Age in years, mean (SD) & $75.1(8.4)$ & $-0.98(-1.14,-0.81)^{*}$ \\
\hline Female & $428(70.3)$ & $-1.22(-4.64,2.21)$ \\
\hline Living alone & $236(38.9)$ & $-4.51(-7.71,-1.33)^{\star *}$ \\
\hline Osteoarthritis & $340(55.9)$ & $-8.57(-11.65,-5.50)^{*}$ \\
\hline Stroke & $81(13.3)$ & $-12.20(-16.70,-7.70)^{\star}$ \\
\hline Cardiovascular disease & $200(32.9)$ & $-7.66(-10.77,-4.55)^{\star}$ \\
\hline Respiratory disease & $134(22.0)$ & $-6.23(-9.98,-2.49)^{\star \star}$ \\
\hline Diabetes mellitus & $106(17.4)$ & $-7.97(-12.04,-3.89)^{\star}$ \\
\hline Parkinson's disease & $12(2.0)$ & $-20.84(-31.93,-9.74)^{\star}$ \\
\hline Polypharmacy (>3 medications) & $373(61.4)$ & $-15.23(-18.20,-12.25)^{\star}$ \\
\hline Pre-index fall functional impairment & $254(41.8)$ & $-17.29(-20.07,-14.50)^{\star}$ \\
\hline Fall history (falls in the previous year, in addition to the index fall) & $308(50.7)$ & $-7.67(-10.68,-4.66)^{\star}$ \\
\hline Vision impairment & $196(32.2)$ & $-6.05(-9.36,-2.73)^{\star}$ \\
\hline BMl, mean $(S D) \dagger$ & $27.90(5.4)$ & $-0.21(-0.50,0.08)$ \\
\hline Cognitive impairment (AMT <7) & $21(3.5)$ & $-22.60(-30.98,-14.21)^{\star}$ \\
\hline Function: HAP-AAS, mean (SD)† & $44.0(19.2)$ & $0.68(-0.62,-0.74)^{*}$ \\
\hline Mobility/balance: TUG (seconds), median (IQR)† & $12.9(10.2-18.8)$ & $-25.54(-27.00,-22.08)^{*} \ddagger$ \\
\hline Depressive symptoms GDS $15 \geq 6 \dagger$ & $148(24.3)$ & $-12.83(-16.31,-9.35)^{*}$ \\
\hline Falls efficacy: MFES, median (IQR) & $8.9(6.7-9.8)$ & $4.37(3.77,4.97)^{*}$ \\
\hline \multicolumn{3}{|l|}{ Index fall injury type $\dagger$} \\
\hline Fracture, dislocation or internal & $261(43.1)$ & $7.44(4.34,10.55)^{*}$ \\
\hline Sprain or strain & $151(24.8)$ & $3.71(0.12,7.32)^{\star \star}$ \\
\hline Contusion, abrasion or laceration & $404(66.6)$ & $-2.96(-6.26,0.35)$ \\
\hline \multicolumn{3}{|l|}{ 12-month follow-up period data } \\
\hline 0 falls & $308(50.7)$ & 1.00 \\
\hline 1 fall with no serious injury & $110(18.1)$ & $-4.43(-8.45,-0.41)^{\star \star}$ \\
\hline 2 or more falls with no serious injury & $145(23.9)$ & $-12.64(-16.26,-9.02)^{\star}$ \\
\hline 1 or more falls with a serious injury & $45(7.4)$ & $-22.64(-28.43,-16.84)^{\star}$ \\
\hline Intervention group allocation & $296(48.9)$ & $-0.22(-3.36,2.91)$ \\
\hline \multicolumn{3}{|l|}{ Outcome measure } \\
\hline 12-month follow-up function: HAP-AAS, mean (SD) & $48.1(19.6)$ & \\
\hline
\end{tabular}

${ }^{\star} P<0.001 ;{ }^{\star \star} P<0.05$. †Missing data: BMl: three participants were unable to have their BMI measured, two participant declined to have their BMI measured and one data point was missing HAP-AAS: one participant declined to answer; TUG: four participants were unable to perform the TUG, two participants declined to perform the TUG and one data point was missing; GDS 15: three participants were unable to have their GDS 15 assessed, two participants declined to answer; Index fall leg injury: up to four points were not recorded adequately for coding. $\ddagger$ log transformation taken. ADL, acts of daily living; AMT, Abbreviated Mental Test; BMI, body mass index; Cl, confidence interval; HAP-AAS, Human Activity Profile Adjusted Activity Score; IQR, interquartile range; MFES, Modified Falls Efficacy Scale; SD, standard deviation; stroke; TUG, Timed Up and Go.

variable (TUG) or dichotomising (MFES) if no suitable transformation was found. Analyses were performed with data from the participants completing the 12-month follow-up assessment from within the RCT, with adjustment for the allocation to intervention or control group.

Baseline assessment, index fall injury data and 12-month follow-up period factors (falls and injuries over the 12-month follow-up period) were entered into a multivariable linear regression analysis and those factors significantly associated with the outcome, as measured with the likelihood ratio test, were retained in the final regression model. Regression diagnostics indicated that regression assumptions were satisfied.

\section{Results}

Description of the study sample

From the 712 participants assessed at baseline, 610 (85.7\%) participants completed the follow-up at 12 months (Figure 1). The 102 people who did not complete the 12 -month assessment were significantly more likely than those continuing in the study to be cognitively impaired or depressed, have more medical conditions, have a slower TUG time, a lower MFES and require assistance with ADLs at the baseline assessment. Two further participants completed the 12-month follow-up assessments but did not have HAP data available (one unable to complete, one refused to complete).

The characteristics of the 608 participants included in the final sample for this study are shown in Table 1 . The mean age of the participants was 75.1 years (standard deviation (SD) \pm 8.4$)$ and $428(70.3 \%)$ were female.

The mean HAP-AAS at the baseline assessment was 44.0 (95\% CI 42.5, 45.6), indicating that the average participant could walk two blocks on level ground but not change the sheets on the bed or sweep for 5 minutes without stopping [25]. From the baseline assessment to the 12-month follow-up assessment, the HAP-AAS mean score improved by $4.1(95 \%$ CI $2.8,5.3)$ and $37.3 \%(95 \%$ CI $33.4,41.2)$ of participants declined in their HAP-AAS scores. The mean HAP-AAS at the 12-month follow-up assessment was 48.1 (95\% CI 46.5, 50.0), indicating that the average participant could change the sheets on the bed, in addition to walking 
Figure 1: Flow diagram.

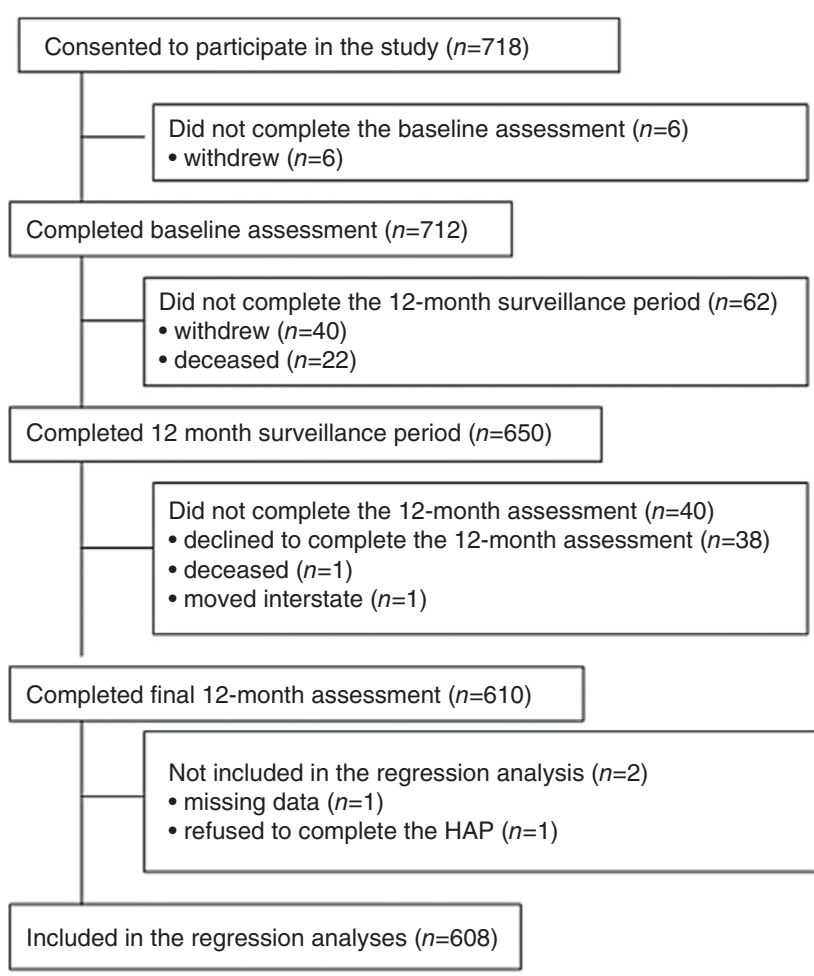

one to two blocks on level ground, but not sweep for 5 minutes without stopping [25].

\section{Univariate linear regression analyses}

Many factors were predictive of function one year after the index fall (Table 1). Gender, allocation arm in the RCT and the index fall injury type had no association with HAP-AAS at the 12-month follow-up assessment.

\section{Multivariable regression analysis}

From the baseline assessment, increased age, the self-reported presence of osteoarthritis or diabetes, polypharmacy, preindex fall functional impairment, falls history, cognitive impairment, taking a longer time to complete the TUG at baseline and having lower falls efficacy were predictive of lower function (HAP-AAS score) at the 12-month follow-up assessment (Table 2). From the 12-month follow-up period, sustaining falls and severe injuries were predictive of lower function (HAP-AAS). Having higher function (HAP-AAS score) at the baseline assessment was predictive of better function at the 12-month follow-up assessment. The (transformed) TUG score was associated with HAP-AAS at the 12-month follow-up assessment.

\section{Discussion}

This study demonstrated that approximately two-thirds of participants at 12 months post-fall improved from their baseline post-fall functional levels and one-third worsened. From the baseline assessment increased age, having osteoarthritis,

\section{Table 2: Multivariable regression analyses of the predictors of long term function, measured by the HAP-AAS, at the 12-month follow-up assessment}

\begin{tabular}{lc}
\hline & $\begin{array}{c}\text { Effect on function (HAP-AAS) at } \\
\text { 12-month follow-up }(95 \% \mathrm{Cl}) \\
n=596 \dagger\end{array}$ \\
\hline Age in years & $-0.52(-0.60,-0.36)$ \\
Osteoarthritis & $-2.18(-4.16,-0.19)$ \\
Diabetes mellitus & $-4.17(-6.77,-1.57)$ \\
Polypharmacy (>3 medications) & $-3.15(-5.31,-1.00)$ \\
Cognitive impairment (AMT <7) & $-8.30(-13.60,-3.01)$ \\
Pre-index fall functional impairment & $-5.29(-7.61,-2.97)$ \\
Falls history (one or more falls in the previous & $-2.10(-4.11,-0.09)$ \\
$\quad 12$ months, in addition to the index fall) & \\
Function at baseline: HAP-AAS (score) & $0.32(0.25,0.40)$ \\
Mobility/balance at baseline: TUG (seconds) & $-6.54(-9.26,-3.82)$ \\
Falls efficacy at baseline: MFES (<8.6) & $-3.32(-5.60,-1.03)$ \\
12-month follow-up period & \\
$\quad 0$ falls & 1.00 \\
1 fall with no serious injury & $-2.79(-5.42,-0.17)$ \\
2 or more falls with no serious injury & $-4.99(-7.47,-2.50)$ \\
1 or more falls with a serious injury & $-9.69(-13.57,-5.79)$ \\
Intervention group allocation & $-0.52(-2.43,1.39)$ \\
\hline
\end{tabular}

†Missing data: HAP-AAS: one participant declined to answer; TUG: four participants were unable to perform the TUG, two participants declined to perform the TUG and one data point was missing; Index fall leg injury: four points were not recorded adequately for coding. łlog transformation. AMT, Abbreviated Mental Test; Cl, confidence interval; HAP-AAS, Human Activity Profile Adjusted Activity Score; MFES, Modified Falls Efficacy Scale; TUG, Timed Up and Go test.

having diabetes, polypharmacy, pre-index fall functional impairment, falls history, being cognitively impaired, taking a longer time to complete the TUG, having lower falls efficacy and lower function at baseline were predictive of lower function (HAP-AAS score) at the 12-month follow-up assessment. The injury type sustained in the index fall had no effect on function at 12 months. Sustaining falls and severe injuries in the 12-month follow-up period was also a strong predictor of lower function at the 12-month follow-up assessment.

This is the first study to investigate function one year after a fall and presentation to an ED. In comparison to previous studies looking at function over three months or less $[10,26]$, this current study found pre-index fall function, falls history and medical conditions were risk factors for lower function at the time of follow-up, rather than the fall injury itself. These findings are supported by the study that measured function at six months post-index fall [12]. These results indicate that, in addition to initial injury management and short-term service provision in the ED, it is important to consider pre-existing conditions such as preindex fall function and fall history when looking an older person's likelihood of long-term recovery.

An additional implication of these findings is a potential good news story, for the majority of older people sustaining a fall and injury and presenting to an ED; that although initially debilitating, there was no evidence that injury had an impact on function for the majority of participants one year on. This finding is supported by previous studies that have found older functionally independent people often experience a 
period of disability and then generally regain their level of function $[27,28]$. However, these studies have also found that periods of disability were risk factors for further disability events and that 'recovery from disability... is often shortlasting' [27]. This current ED-based study and previous population based studies highlight the importance of recognising older people at risk of recurrent loss of function and considering preventative and rehabilitative services to maintain functional independence.

Notably, the $7 \%$ of participants sustaining one or more fall-related serious injuries in the 12-month follow-up period scored 9 points lower on the HAP-AAS at the 12-month follow-up, compared to those not falling and sustaining serious injuries, after adjusting for other factors. This difference in function between non-fallers and fallers sustaining an injury is larger than the HAP-AAS score of 6.8 , which has been found to be 'required to be $90 \%$ confident that change is beyond measurement error' [18]. The effect of falls and injuries on function has been previously studied $[7,8]$; this study adds to this body of evidence and highlights the importance of identifying those at risk of loss of independence and also intervening to prevent further falls and episodes of loss of function within this population.

An unusual finding in this study was that sustaining a fracture in the index fall was associated with better function at the 12-month follow-up in the univariate analysis. We hypothesise that this finding is related to the reasons an older person may go to an ED after a fall. Less independent older people may present to an ED after a fall because of a minor injury compounding already present limitations (e.g. unable to rise from the floor after a fall). However, independent older people would only be likely to present to an ED with more severe injures/fractures and would tend to manage any minor injuries themselves or through their primary care providers. Hence, in this study, the participants sustaining fractures are more likely to have better function. Our hypothesis is supported by the outcomes of the multifactorial analysis, as the association between fracture and better function at 12 months disappears when other factors (e.g. pre-index fall functional impairment) were adjusted for.

Three of the baseline assessment factors (falls history, preindex fall function, impaired mobility/balance) predictive of lower levels of function at the 12-month follow-up have been previously found to be the factors most strongly predictive of falls in the same population over 12 months [29]. In this previous study, these factors were combined to form the FROP-Com screen, which has been successfully implemented as a screening tool for falls in EDs [14]. This new finding that the factors were also predictive of function at 12 months has the important clinical implication that ED staff could potentially use the same screening tool to identify people at risk of falls and at risk of lower levels of function in the longer term.

There are limitations to this study. Firstly, pre-index fall function was self-reported and the baseline assessment took place on average 3 weeks after the ED presentation. Secondly, there was a loss of $14 \%$ of participants due to drop outs and death. In the comparison of HAP-AAS at baseline to follow-up, if those lost declined in function there may be an overestimation in the percentage of participants improving. In the investigation of predictors of function, the losses are likely to result in an underestimation of any associations in this study compared to the true underlying associations. A further limitation is that co-morbidities during the 12-month follow-up period were not recorded. The strengths of this study were its longitudinal approach, large sample size and that the sample was generally representative of the older population [15].

In practice, this study highlights the importance of identifying those at high risk of falls post ED discharge, so that those at risk can be identified and potentially beneficial interventions can be put in place and further falls and subsequent injuries prevented. Although fall prevention interventions have been found to be effective in preventing falls after an ED visit [30], numerous studies have demonstrated that there are few fall prevention interventions being implemented for older people who have sustained a fall and presented to the ED $[13,31,32]$. An additional important clinical finding of this research is that some of the factors predictive of decreased function at 12 months have been previously identified as the factors most predictive of falls over the 12-month follow-up period [29]. This indicates that single screening tools, such as the FROP-Com screen [29], may be used to identify those most likely to have negative outcomes of falls and lower long-term function in this population.

In summary, the majority of older people who have fallen presenting to the ED after a fall improved in their levels of function 12 months after thier post-ED baseline assessment, and the index fall and injury had no effect on function at 12 months. However, one-third worsened over the 12 months, and those with lower function at 12 months were those with lower levels of function, medical conditions and a history of falls prior to the index fall and who also experienced ongoing falls and injuries over the 12-month follow-up period. These findings highlight the importance of recognising older people at risk of further falls and recurrent loss of function in the ED and then intervening to maintain independence and prevent further falls, in addition to initial injury management.

\section{Acknowledgements}

The Australian Government Department of Veterans' Affairs and the Victorian Department of Human Services. We thank the participants in the Falls Aren't Us Study; the National Ageing Research Institute staff: Leslie Dowson, Sarah Tarquinio, and Marcia Fearn, research assistants; Sue Williams and Sally A'Beckett, research physiotherapists; Terry Sullivan and Fiona Bremnar, research occupational therapists; the Falls Aren't Us steering committee. 


\section{Key Points}

- The majority of older people who presented to the ED after a fall improved in their levels of function 12 months after their post-ED baseline assessment, and the index fall and injury had no effect on function at 12 months.

- Pre-index fall existing conditions, such as osteoarthritis, having diabetes, polypharmacy, and falls history were stronger predictors of poorer function after one year. These factors could be considered in the ED for identifying older people who have fallen who are at risk of further loss of function in the longer term.

- Sustaining further falls and injuries during the 12 months after discharge from the ED was highly predictive of lower function at 12 months. This finding highlights the importance of recognising those at risk of further falls and loss of function, in the ED, with the aim of preventing these ongoing problems.

\section{References}

1 Millán-Calenti JC, Tubío J, Pita-Fernández $S$ et al. Prevalence of functional disability in activities of daily living (ADL), instrumental activities of daily living (IADL) and associated factors, as predictors of morbidity and mortality. Archives of Gerontology and Geriatrics 2010; 50: 306-310.

2 LaFortune G, Balestat G, Disability Study Expert Group Members. Trends in severe disability among elderly people: Assessing the evidence in 12 OECD countries and the future implications [internet]. 2007. [Cited 8 November 2013.] Available from URL: http://www.oecd.org/australia/ 38343783.pdf

3 Turvey CL, Schultz SK, Beglinger L, Klein DM. A longitudinal communitybased study of chronic illness, cognitive and physical function, and depression. The American Journal of Geriatric Psychiatry 2009; 17: 632-641.

4 Gaugler JE, Duval S, Anderson KA, Kane RL. Predicting nursing home admission in the U.S: A meta-analysis. BMC Geriatrics 2007; 7: 13.

5 Kiel DP, O'Sullivan P, Teno JM, Mor V. Health care utilization and functional status in the aged following a fall. Medical Care 1991; 29: 221228.

6 Sui X, Laditka JN, Hardin JW, Blair SN. Estimated functional capacity predicts mortality in older adults. Journal of the American Geriatrics Society 2007; 55: 1940-1947.

7 Gill TM, Allore HG, Holford TR, Guo Z. Hospitalization, restricted activity, and the development of disability among older persons. JAMA: the Journal of the American Medical Association 2004; 296: 2115-2124.

8 Tinetti M, Williams C. The effect of falls and fall injuries on functioning in community-dwelling older persons. The Journals of Gerontology. Series A, Biological Sciences and Medical Sciences 1998; 53: M112M119.

9 Close JC, Lord SR, Antonova EJ et al. Older people presenting to the emergency department after a fall: A population with substantial recurrent healthcare use. Emergency Medicine Journal 2012; 29: 742-747.

10 Russell MA, Hill KD, Blackberry I, Day LL, Dharmage SC. Falls risk and functional decline in older fallers discharged directly from emergency departments. The Journals of Gerontology. Series A, Biological Sciences and Medical Sciences 2006; 61A: 1090-1095.
11 Shapiro MJ, Partridge RA, Jenouri I, Micalone M, Gifford D. Functional decline in independent elders after minor traumatic injury. Academic Emergency Medicine 2001; 8: 78-81.

12 Bloch F, Jegou D, Dhainaut JF et al. Do ED staffs have a role to play in the prevention of repeat falls in elderly patients? The American Journal of Emergency Medicine 2009; 27: 303-307.

13 Salter $\mathrm{AE}$, Khan KM, Donaldson MG et al. Community-dwelling seniors who present to the emergency department with a falls do not receive guideline care and their risk profile worsens significantly: A 6-month prospective study. Osteoporosis International 2006; 17: 672-683.

14 Waldron N, Dey I, Nagree Y, Xiao J, Flicker L. A multi-faceted intervention to implement guideline care and improve quality of care for older people who present to the emergency department with falls. BMC Geriatrics 2011; $11: 6$.

15 Russell MA, Hill KD, Day LM, Blackberry I. A randomized controlled trial of a multifactorial falls prevention intervention for older fallers presenting to Emergency Departments. Journal of the American Geriatrics Society 2010; 58: 2265-2274.

16 Russell MA, Hill KD, Blackberry I, Day LM, Dharmage SC. The reliability and predictive accuracy of the falls risk for older people in the community assessment (FROP-Com) tool. Age and Ageing 2008; 37: 634-639.

17 Hodkinson $H$. Evaluation of a mental test score for assessment of mental impairment in the elderly. Age and Ageing 1972; 1: 233-238.

18 Davidson M, de Morton N. A systematic review of the Human Activity Profile. Clinical Rehabilitation 2007: 21: 151-162.

19 Podsiadlo D, Richardson S. A test of basic functional mobility for frail elderly persons. Journal of the American Geriatrics Society 1991; 38: 142-148.

20 Sheikh J, Yesavage J. Geriatric depression scale (GDS). Recent evidence and development of a shorter version. Clinical Gerontologist 1986; 5: 165-173.

21 Hill KD, Schwarz JA, Kalogeropoulos AJ, Gibson SJ. Fear of falling revisted. Archives of Physical Medicine and Rehabilitation 1996; 77: 1025-1029.

22 Hill K, Womer M, Russell M, Blackberry I, McGann A. Fear of falling in older fallers presenting at emergency departments. Journal of Advanced Nursing 2010; 66: 1769-1779.

23 The Association for the Advancement of Automotive Medicine. The Abbreviated Injury Scale: 1990 Revision: Update 98, 2001: Barrington, IL: Association for the Advancement of Automatic Medicine.

24 Tinetti M, Williams C. Falls, injuries due to falls, and the risk of admission to a nursing home. The New England Journal of Medicine 1997; 337: 1279-1284.

25 Fix A, Daughton D. Human Activity Profile. Professional Manual. Lutz, FL: Psychological Assessment Resources, 1988.

26 Grisso JA, Schwarz DF, Wolfson V, Polansky M, LaPann K. The impact of falls in an inner-city elderly African-American population. Journal of the American Geriatrics Society 1992; 40: 673-678.

27 Hardy SE, Gill TM. Recovery from disability among community-dwelling older persons. JAMA: the Journal of the American Medical Association 2004; 291: 1596-1602.

28 Hardy SE, Dubin JA, Holford TR, Gill TM. Transitions between states of disability and independence among older persons. American Journal of Epidemiology 2005; 161: 575-584.

29 Russell MA, Hill KD, Day LM, Blackberry I, Gurrin LC, Dharmage SC. Development of the Falls Risk for Older People in the Community (FROPCom) screening tool. Age and Ageing 2009; 38: 40-46.

30 Close J, Ellis M, Hooper R, Glucksman E, Jackson S, Swift C. Prevention of falls in the elderly trial (PROFET): A randomised controlled trial. Lancet 1999; 353: 93-97.

31 Miller E, Wightman E, Rumbolt K et al. Management of fall-related injuries in the elderly: A retrospective chart review of patients presenting to the emergency department of a community-based teaching hospital. Physiotherapy Canada. Physiotherapie Canada 2009; 61: 26-37.

32 Naughton $\mathrm{C}$, McGrath E, Drennan $\mathrm{J}$ et al. The profile and follow-up of patients who attend the emergency department following a fall. International Emergency Nursing 2011. [Cited 8 November 2013.] Available from URL: http://www.sciencedirect.com/science/article/pii/ S1755599X11000735 